\title{
Multi-Task and Multi-Corpora Training Strategies to Enhance Argumentative Sentence Linking Performance
}

\author{
Jan Wira Gotama Putra ${ }^{\dagger}$, Simone Teufel ${ }^{\ddagger \dagger}$, Takenobu Tokunaga ${ }^{\dagger}$ \\ ${ }^{\dagger}$ Tokyo Institute of Technology, Japan \\ $\ddagger$ University of Cambridge, United Kingdom \\ \{gotama.w.aa@m, take@c\}.titech.ac.jp, simone.teufelecl.cam.ac.uk
}

\begin{abstract}
Argumentative structure prediction aims to establish links between textual units and label the relationship between them, forming a structured representation for a given input text. The former task, linking, has been identified by earlier works as particularly challenging, as it requires finding the most appropriate structure out of a very large search space of possible link combinations. In this paper, we improve a state-of-the-art linking model by using multi-task and multi-corpora training strategies. Our auxiliary tasks help the model to learn the role of each sentence in the argumentative structure. Combining multi-corpora training with a selective sampling strategy increases the training data size while ensuring that the model still learns the desired target distribution well. Experiments on essays written by English-as-a-foreign-language learners show that both strategies significantly improve the model's performance; for instance, we observe a $15.8 \%$ increase in the F1-macro for individual link predictions.
\end{abstract}

\section{Introduction}

Argument mining (AM) is an emerging area that addresses the automatic analysis of argumentation. Many recent studies commonly try to tackle two major tasks (Lawrence and Reed, 2020). The first of these is argumentative component identification, in which argumentative units (ACs) and nonargumentative components (non-ACs) including their boundaries are determined. ACs can be further classified according to their role in argumentation, e.g., major claim, ${ }^{1}$ claim and premise (Stab and Gurevych, 2017). The second task is called argumentative structure prediction, which first establishes links from source to target ACs (this is called the linking task) and then labels the relationship between them, for instance using the support

\footnotetext{
${ }^{1}$ The major claim is the statement expressing the writer's view on the discussion topic; also called main stance or main claim.
}

and attack relation labels (this is called the relation labelling task) (Stab and Gurevych, 2017).

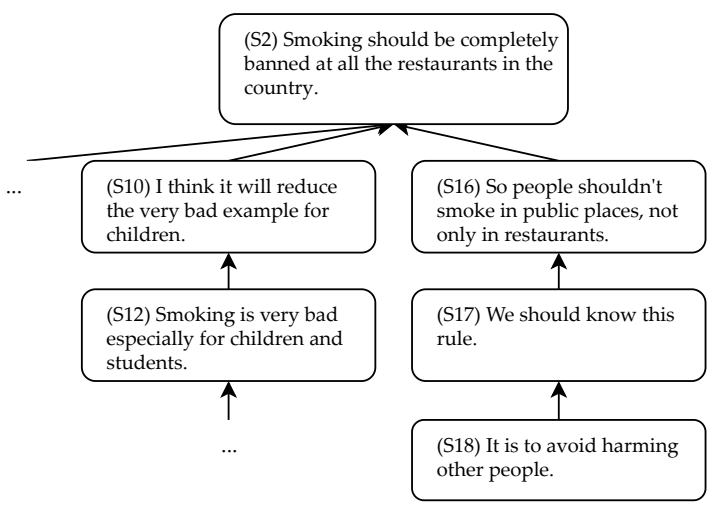

Figure 1: Illustration of linking task, using part of an essay discussing the topic "Smoking should be banned at all restaurants in the country."

The linking task has been identified by earlier works as particularly challenging (Lippi and Torroni, 2016; Cabrio and Villata, 2018; Lawrence and Reed, 2020). There are many possible combinations of links between textual units, and a linking model has to find the most proper structure out of a very large search space. Another typical challenge in AM is the size of annotated corpora (Schulz et al., 2018). Corpus construction is a complex and time-consuming process; it also often requires a team of expert annotators. Existing corpora in AM are relatively "small" compared with more established fields, such as machine translation or document classification. This hinders training AM models when using a supervised machine learning framework.

In this paper, we perform the linking task for essays written by English-as-a-foreign-language (EFL) learners. Given an essay, we identify links between sentences, forming a tree-structured representation of argumentation in the text. Figure 1 illustrates the task. Our contributions are twofold. First, we propose two structural-modelling related 
auxiliary tasks to train our model in a multi-task learning (MTL) fashion. Past studies commonly executed several AM tasks or using discourse and rhetorical auxiliary tasks in the MTL setting (cf. Section 2). In comparison with other works, our auxiliary tasks are advantageous in that they do not require any additional annotation. Second, we combine multi-corpora training with a selective sampling strategy to increase the training data size. We explore the use of mixed training data of in-domain EFL and out-domain ${ }^{2}$ student-essay corpora that were annotated using different schemes and of different quality, while ensuring that the model still learns the properties of the target in-domain data well.

The choice of EFL texts in this study aims to contribute to a less attended area. In AM, it is common to use well-written texts by proficient authors (e.g., Ashley, 1990; Peldszus and Stede, 2016). However, student texts often suffer from many problems because they are still in the learning phase. Even more, EFL texts are also less coherent and less lexically rich, and exhibit less natural lexical choices and collocations (Silva, 1993; Rabinovich et al., 2016). There are more non-native English speakers (Fujiwara, 2018), and yet, to the best of our knowledge, only one preceding study in AM concerned the EFL genre (Putra et al., 2021b). The codes accompanying this paper are publicly available. ${ }^{3}$

\section{Related Work}

\subsection{Argumentative Structure Prediction}

A variety of formulations have been proposed for the linking task. Traditional approaches formulated it as a pairwise classification task, predicting whether an argumentative link exists between a given pair of ACs (Stab and Gurevych, 2014). A further post-processing step can also be performed to combine the local predictions into an optimised global structure, e.g., using the minimum-spanningtree algorithm (Peldszus and Stede, 2015). Recent studies proposed a more global approach instead, considering the entire input context. For instance, Potash et al. (2017) formulated the linking task as a sequence prediction problem. They jointly performed $\mathrm{AC}$ classification and $\mathrm{AC}$ linking at once,

\footnotetext{
${ }^{2}$ Past studies used the term domain in a broad context. It has at least five different senses: text genre, text quality, annotation scheme, dataset and topic (or prompt). In the rest of this paper, we use the specific meaning whenever possible.

${ }^{3}$ https: / / github.com/wiragotama/ ArgMin2021
}

assuming that the segmentation and $\mathrm{AC}$ vs non- $\mathrm{AC}$ categorisation have been pre-completed. They experimented on the microtext corpus (Peldszus and Stede, 2016) and the persuasive essay corpus (PEC, Stab and Gurevych (2017)).

Eger et al. (2017) formulated argumentative structure parsing in three ways: as relation extraction, as sequence tagging and as dependency parsing tasks. They defined a BIO tagging scheme and performed end-to-end parsing at token-level, executing all subtasks (i.e., segmentation, unit type classification, linking and relation labelling) at once. Ye and Teufel (2021) also performed endto-end parsing at the token-level. They proposed a more efficient representation for the dependency structure of arguments, and achieved the state-ofthe-art performance for component and relation identifications on the PEC using a biaffine attention model (Dozat and Manning, 2017).

The biaffine attention model was originally designed to parse token-to-token dependency, but Morio et al. (2020) extended it to parse proposition (segment) level dependency. Their model dealt with graph-structured arguments in the Cornell eRulemaking corpus (Park and Cardie, 2018). Using the same architecture, Putra et al. (2021b) parsed tree-structured EFL essays in the ICNALE-AS2R corpus (Ishikawa, 2013, 2018; Putra et al., 2021a,b). In tree-structured argumentation, it is common for groups of sentences about the same sub-topic to operate as a unit, forming a sub-tree (sub-argument). Putra et al. (2021b) found that their linking model has problems in constructing sub-trees, that is, it splits a group of sentences that should belong together into separate sub-arguments (sub-trees) or, conversely, groups together sentences that do not belong together into the same sub-trees.

\subsection{Low-Resource and Cross-Domain Argument Mining}

Several approaches have been applied to alleviate the data sparsity problem in AM. Al-Khatib et al. (2016) used a distant supervision technique to acquire a huge amount of data without explicit annotation. Accuosto and Saggion (2019) pre-trained a discourse parsing model and then fine-tuned it on AM tasks. Lauscher et al. (2018) investigated the MTL setup of argumentative component identification and rhetorical classification tasks. Schulz et al. (2018) performed a cross-genre argumenta- 
tive component identification. They employed a sequence tagger model with a shared representation but different prediction layers for each genre.

Data augmentation can also be applied to mitigate the data sparsity problem. This aims to increase the amount of training data without directly collecting more data (Liu et al., 2020; Feng et al., 2021). A relatively straightforward strategy is to use multiple corpora when training models. For example, Chu et al. (2017) proposed a mixed fine-tuning approach for machine translation; they trained a model on an out-genre corpus and then fine-tune it on a dataset that is a mix of the targetgenre and out-genre corpora. However, the use of multiple corpora of different genres is challenging in AM because argumentation is often modelled differently across genres (Lippi and Torroni, 2016; Lawrence and Reed, 2020). Daxenberger et al. (2017) found that training a claim identification model with mixed-genre corpora only perform as good as training on each specific corpus. The use of data augmentation may cause the distributional shift problem as well, where the augmented data alter the target distribution that should be learned by the model (Feng et al., 2021).

Putra et al. (2021b) provided some insights on the possible conditions for multi corpora training by experimenting using texts of different quality. They trained argumentative structure prediction models on EFL essays (in-domain) and parallel improved version of the texts (out-domain), and evaluated on the EFL texts. Their cross-domain system attained $94 \%$ accuracy of end-to-end indomain performance. This signals the potential to use corpora of different text quality altogether to train a parsing model, as long as the text genre stays the same. However, mixed-quality corpora training has yet to be tried in practice.

In recent years, there has also been a growing interest towards more generic AM models. For example, Stab et al. (2018) proposed a simple annotation scheme for argument retrieval that is applicable in heterogeneous sources and can be performed by untrained annotators. Cocarascu et al. (2020) explored various deep learning architectures and features that work well across various datasets for the relation labelling task. They also provided a comparison of using contextualised and non-contextualised embeddings for the task. On the other hand, Ruiz-Dolz et al. (2021) compared the performance of transformer-based language mod- els on the cross-topic relation labelling task.

\section{Dataset}

Our target texts are sourced from the ICNALE-AS2R, a corpus of 434 essays written by Asian college students with intermediate proficiency. ${ }^{4}$ There are 6,021 sentences in total with 13.9 sentences on average per essay. To the best of our knowledge, this is the only currently publicly-available AM corpus focusing on EFL texts. The corpus is annotated at sentence-level, that is, a sentence corresponds to one argumentative unit. The corpus differentiates sentences as ACs (5,799 sents.) and non-ACs (222 sents.), without further classification of AC types. Links are established between ACs to form tree structure (avg. depth of 4.3, root at depth 0 in the corpus), where the major claim acts as the root. Four relation labels are employed to label the links: support, attack, detail and restatement.

\section{Proposed Method}

In this work, we experiment on the linking task. Given an entire essay of $N$ sentences as input, $s_{1}, \ldots, s_{N}$, a linking model outputs the distance $d_{1}, \ldots, d_{N}$ between each sentence $s_{i}$ to its target. For instance, if a sentence is connected to its preceding sentence, the distance is $d=-1$. We consider those sentences that have no explicitly annotated outgoing links as linked to themselves $(d=0)$; this concerns major claims (roots) and non-ACs. We do not consider labelling the links with their relationships in this paper.

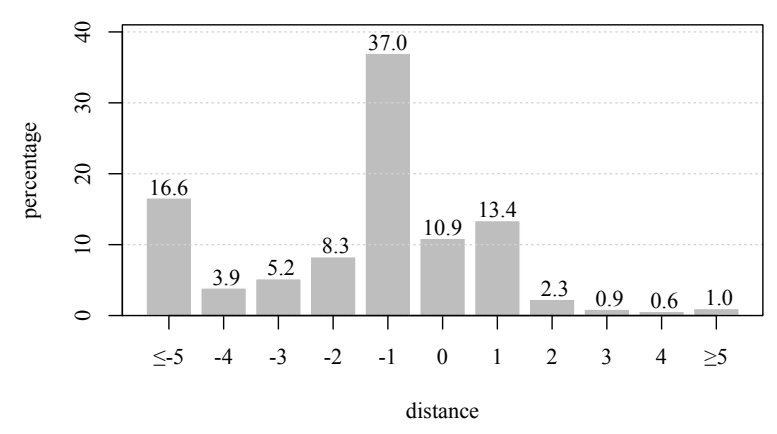

Figure 2: Distribution of distance (in percent) between related sentences in the ICNALE-AS2R corpus.

Figure 2 shows the distance distribution between the source and target sentences in the

\footnotetext{
${ }^{4}$ https://www.gsk.or.jp/en/catalog/ gsk2021-a. The approximated CEFR level of the essay authors are A2 (94 essays), B1 (253) and B2 (87).
} 
ICNALE-AS2R corpus, ranging $[-26, \ldots,+15]$. Adjacent links predominate $(50.4 \%)$. Short distance links $(2 \leq|d| \leq 4)$ make up $21.2 \%$ of the total. Backward long distance links at $d \leq-5$ are $16.6 \%$, whereas forward long distance links are rare $(1.0 \%)$. Self-loops make up $10.9 \%$ of the total. Following recent advances in AM, we approach the linking task as a sentence-to-sentence dependency parsing.

\subsection{Baseline}

We use Putra et al.'s (2021b) architecture as the state-of-the-art baseline ("BIAF" model, cf. Figure 3). In this architecture, input sentences are first encoded into their respective sentence embeddings using sentence-BERT encoder (Reimers and Gurevych, 2019). The resulting sentence embeddings are then passed into a dense layer for dimensionality reduction. The results are then fed into a bidirectional long-short-term memory network $($ BiLSTM) layer $(\# s t a c k=3$ ) to produce contextual sentence representations. These representations are then passed into two different dense layers to encode the corresponding sentence when it acts as a source $\left(h^{\text {(source) }}\right)$ or target $\left(h^{\text {(target) }}\right)$ in a relation.

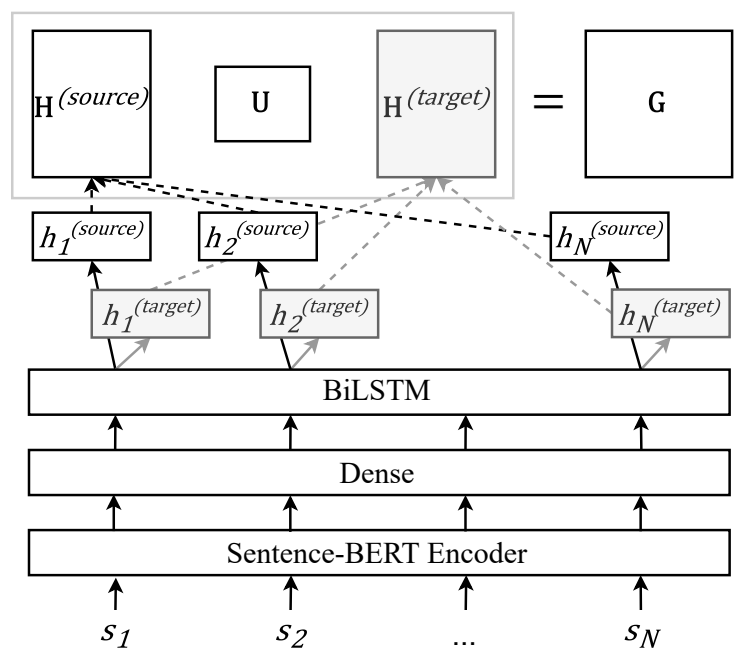

Figure 3: Biaffine attention model (BIAF).

Finally, a biaffine transformation (Dozat and Manning, 2017) is applied to all source and target representations to produce the final output matrix $\mathbf{G} \in \mathbb{R}^{N \times N}$, where each cell $g_{i, j}$ represents the probability (or score) of the source sentence $s_{i}$ pointing to $s_{j}$. Equation (1) and (2) show the biaffine transformation $(f)$, where $\mathbf{U}$ and $\mathrm{W}$ are weight matrices and $b$ is bias.

$$
\begin{aligned}
f\left(x_{1}, x_{2}\right) & =x_{1}{ }^{\mathrm{T}} \mathbf{U} x_{2}+\mathbf{W}\left(x_{1} \oplus x_{2}\right)+b \\
g_{i, j} & =f\left(h_{i}^{(\text {source })}, h_{j}^{\text {(target })}\right)
\end{aligned}
$$

The Chu-Liu-Edmonds algorithm (Chu and Liu, 1965; Edmonds, 1967) is applied to create a minimum spanning tree out of the matrix $\mathbf{G}$. The links in the tree are then converted into distance predictions between source and target sentences for evaluation purpose.

\subsection{Multi-Task Learning with Structural Signal}

We propose to extend the BIAF model in an MTL setup using two novel structural-modelling-related auxiliary tasks.

The first auxiliary task is a quasi argumentative component type (QACT) prediction. ICNALE-AS2R corpus does not assign AC types per se, but we can compile the following four sentence types from the tree typology:

- major claim (root): only incoming links,

- $A C$ (non-leaf): both outgoing and incoming links,

- $A C$ (leaf): only outgoing links and

- non-AC: neither incoming nor outgoing links.

For example, S2 is the major claim of Figure 1, S10 is AC (non-leaf) and S17 is AC (leaf). The QACT prediction task should help the linking model to learn the role of sentences in the argumentative structure, as well as the property of links for each sentence. This auxiliary task improved a sequencetagger based linking model in Putra et al. (2021b), but it has not been applied to the BIAF model yet.

The second auxiliary task concerns node depth (ND) prediction. There are six depth categories employed in this paper: depth 0 to depth 4 , and depth 5+. The argumentative structure in ICNALE-AS2R corpus is hierarchical, and there is no relations between nodes (sentences) at the same depth. The ND prediction task should help the model to learn the placement of sentences in the hierarchy and guide where each sentence should point at, that is, sentences at depth $X$ point at sentences at depth $X-1$.

We also propose to use sentence position (spos) embedding as an input feature because it has been proved to be useful in other studies (e.g., Song et al., 2020). The sentence position encoding is 


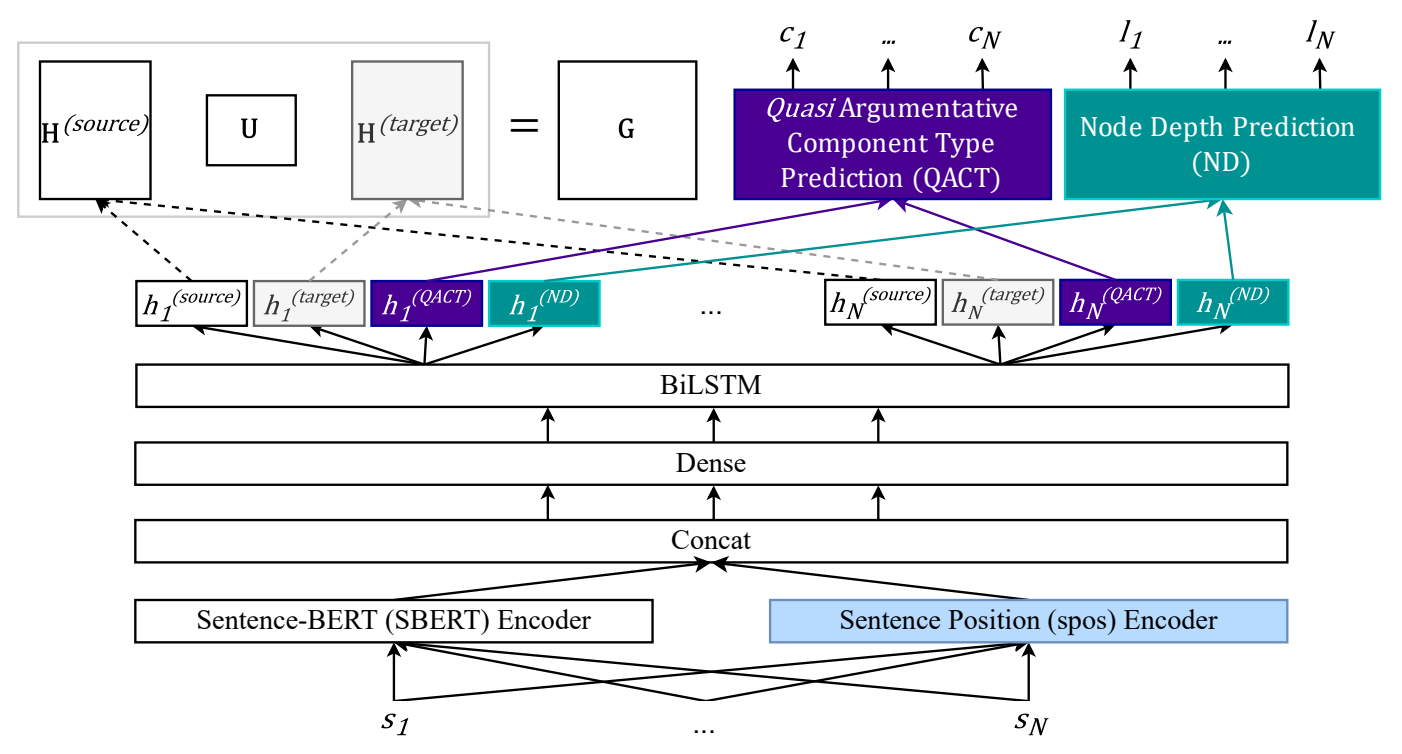

Figure 4: Our proposed extension for the BIAF model. Newly added modules are coloured.

calculated by dividing a sentence position by the essay length. When using this feature, sentence embeddings and spos encoding are concatenated before being passed to the first dense layer. Figure 4 illustrates our proposed architecture.

\subsection{Multi-Corpora Training}

In this paper, we also consider the use of multicorpora training strategy, employing essays in the PEC as additional training data. Both ICNALE-AS2R and PEC provide argumentative essays written by students (in English) and represents argumentative structures as trees. However, there is no information on the essay authors proficiency (L1 or L2) nor the observed quality of the PEC. Hence, these two corpora might be of different quality. The writing prompts of ICNALE-AS2R and PEC essays are also different.

There are two settings when training using multiple corpora. The first is to use the entire 402 essays in the PEC on top of ICNALE-AS2R train set (" $[\mathrm{P}+\mathrm{I}]$ " setting). However, PEC and ICNALE-AS2R are different in terms of length and annotation scheme. The PEC essays have 18.2 sentences on average (15.1 ACs and 3.1 non-ACs), while ICNALE-AS2R essays have 13.9 sentences on average (13.4 ACs and 0.5 non-ACs). The difference in non-ACs proportion between these corpora is likely caused by the difference in the set of relation labels employed. The essays in PEC were annotated using two relation types: support and attack, while ICNALE-AS2R additionally employed detail and restatement. Hence, there is arguably more information in the ICNALE-AS2R corpus. Particularly, some sentences that should have been annotated as non-ACs (hence not linked to other sentences) in the PEC's scheme might have been annotated as ACs in the ICNALE-AS2R's scheme by using the additional relations.

Due to the differences in annotation scheme and statistical properties, there is a possibility that the model might not properly learn the distribution of ICNALE-AS2R in the $[\mathrm{P}+\mathrm{I}]$ setting. Therefore, we also propose a second selective sampling ("[SS]" setting) strategy to account for the differences between these two corpora. In this setting, we only use PEC essays that are somewhat similar to those of ICNALE-AS2R considering the following two criteria. First, we only use PEC essays having 17 sentences at maximum (ICNALE-AS2R avg. 13.9 + 3.3 SD). Second, the employed PEC essays contain two non-ACs at maximum (ICNALE-AS2R avg. $0.5+0.9 \mathrm{SD}$ ). There are 110 remaining PEC essays after the selective sampling procedure.

Note that ICNALE-AS2R was annotated at the sentence level while PEC was annotated at the segment level. Therefore, we convert the PEC annotation to the sentence level, following the strategy described by Song et al. (2020). If a sentence contains only one $\mathrm{AC}$, we use the whole sentence as an $\mathrm{AC}$; if a sentence contains two or more ACs, we split it into multiple sentences while including the preceding tokens into each AC. Figure 5 illustrates the splitting procedure, where a sentence contain- 
(S1) [To conclude, art could play an active role in improving the quality of people's lives,] (S2) [but I think that governments should attach heavier weight to other social issues such as education and housing needs] (S3)[because those are the most essential ways enable to make people a decent life.]

Figure 5: Illustration of conversion of PEC's segmentlevel annotation to the sentence-level (essay075). Annotated AC segments are written in bold.

ing three ACs is split into three sentences.

\section{Structural Metric}

In this paper, we do not evaluate the model performance only on individual link predictions but also analyse the structural properties of the outputs, giving more insights into the models' ability to learn different aspects of the argumentative structure. We also investigate whether our proposed strategies improve the model performance concerning the grouping of related sentences into the same sub-tree, a challenge to linking models previously (manually) observed in Putra et al. (2021b) (cf. Section 2.1). A metric is needed to quantify the improvement on this aspect, and we propose to use a novel structural metric called $\mathbf{M A R} \mathbf{R}^{\text {dSet }}$, that was developed by Putra et al. (2021a). ${ }^{5}$

Given two structures $A$ and $B$ (i.e., predicted and gold structures), we can quantify their similarity based on the presence of common substructures. We first define a descendant set (dSet) of a node $X$ as the set consisting the node $X$ itself and its descendants. Figure 6 shows examples of dSets (brackets given below the node ID). ${ }^{6}$ For example, the dSet of node- 2 in annotation $A$ of Figure 6 is $\{2,3,4,5\}$. Two corresponding nodes in $A$ and $B$ are required to have identical dSets in order to score a value of 1 . For example, the matching score for node- 2 between annotation $A$ and $B$ in Figure 6 is 0 , while the matching score for node- 3 is 1 . Non-AC nodes are counted as a match if they are deemed non-argumentative in both structures.

With this formulation, we can get a vector $v$ representing the matching scores for all nodes in the two structures. For example, $v=[0,0,1,1,0]$ for Figure 6 . The similarity score between two structures are then calculated as $\mathrm{MAR}^{\mathrm{dSet}}=\frac{\sum_{i=1}^{N} v_{i}}{N}$,

\footnotetext{
${ }^{5}$ MAR stands for Mean Agreement in Recall. Descendant set (dSet) denotes the unit of analysis.

${ }^{6}$ In the implementation, we use sentence position in the input text as node ID.
}

where $N$ denotes the number of nodes; The MAR $^{\mathrm{dSet}}$ score for Figure 6 is 0.4 .

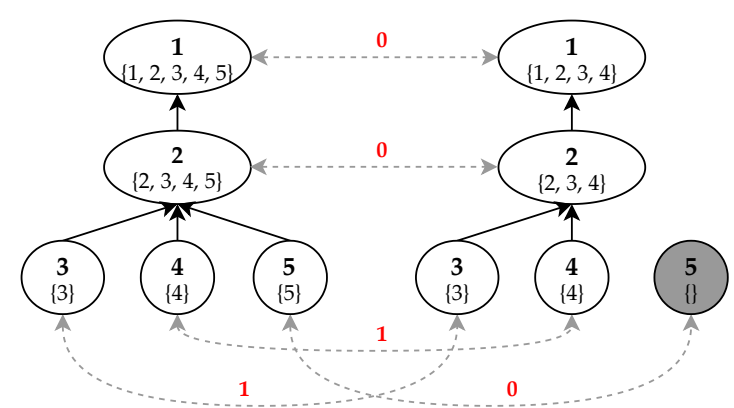

Figure 6: Example of descendant set matching between structure $A$ (left) and $B$ (right). Matching scores are written in red. Grey nodes represent non-AC.

\section{Experiment and Discussion}

\subsection{Experimental Setting}

In this experiment, the ICNALE-AS2R corpus is split into $80 \%$ train set (347 essays , 4,841 sents.) and $20 \%$ test set ( 87 essays, 1,180 sents.). We use the same splits as Putra et al. (2021b). The number of training essays for $[\mathrm{P}+\mathrm{I}]$ and $[\mathrm{SS}]$ settings are 749 (12,162 sents.) and 457 (6,418 sents.), respectively. We run the experiment for 20 times $^{7}$ and report the average performance. Statistical testing, whenever possible, is conducted using the permutation test (Noreen, 1989) on the performance scores of the 20 runs with a significance level of $\alpha=.05$. Here, we also perform an ablation study. Appendix A shows some implementation notes.

\subsection{Quantitative Analysis}

Table 1 shows the experimental results on individual link predictions and MAR ${ }^{\mathrm{dSet}}$. Training the BIAF model using the QACT task improved the performance over the baseline, particularly in terms of F1-macro (non significant difference). In addition, using both QACT+ND auxiliary tasks significantly improved the performance over the baseline in terms of F1-macro. This signals that the proposed MTL setting benefits model performance.

We next look at how spos encoding affects the model performance. Introducing spos to the BIAF+QACT+ND model improves accuracy and $\mathrm{MAR}^{\mathrm{dSet}}$. However, the difference is not significant. Similarly, the difference between

\footnotetext{
${ }^{7}$ Twenty experiments were repeated on the same dataset splits to account for random initialisation in neural networks.
} 


\begin{tabular}{lccc}
\hline Model & Acc. & F1-macro & MAR $^{\text {dset }}$ \\
\hline $\begin{array}{l}\text { Baseline } \\
\text { BIAF }\end{array}$ & .471 & .323 & .419 \\
\hline MTL & & & \\
BIAF+QACT & .473 & .333 & 422 \\
BIAF+QACT+ND & .472 & .338 & 423 \\
\hline Spos & & & \\
BIAF+QACT+spos & .472 & .327 & .421 \\
BIAF+QACT+ND+spos & .475 & .336 & 426 \\
\hline Multi-corpora Training & & & \\
BIAF+QACT+ND [P+I] & .468 & .360 & $\mathbf{. 4 5 5}$ \\
BIAF+QACT+ND [SS] & $\mathbf{. 4 8 9}^{\dagger}$ & $\underline{.374^{\dagger}}$ & $\underline{.452}$ \\
\hline
\end{tabular}

Table 1: Results of individual link predictions (Acc. and F1-macro) and MAR ${ }^{\mathrm{dSet}}$. The best result is shown in bold-face. The $\dagger$ symbol indicates that the difference to the second-best result (underlined) is significant.

BIAF+QACT+spos and BIAF+QACT is not significant. There are two possible explanations for this phenomenon. First, studies in contrastive rhetoric found that non-native speakers tend to structure and organise their texts differently from native speakers (Kaplan, 1966; Silva, 1993). Some students might have used the writing customs and rhetorical strategies of their first language instead of using the common English writing patterns. For example, Asian students sometimes put a claim after its reason, which is not common in Anglo-Saxon cultures (Silva, 1993; Johns, 1986). We observe that some texts in the ICNALE-AS2R corpus are written in the "claim-support" structure but some are written in the "support-claim" structure. Sentences on the same topic are sometimes distantly separated as well. These inconsistencies might have negated the effect of the spos encoding. Second, the output of the BIAF model is a graph $\mathbf{G}$ which considers the directed links between all pairs of sentences. The spos feature might not affect the biaffine transformation much in this context.

The models trained using multiple corpora attain the best performance for individual link and substructure (MAR) predictions. BIAF+QACT+ND $[\mathrm{P}+\mathrm{I}]$ achieves the best performance of .455 in terms of MAR ${ }^{\mathrm{dSet}}$, and BIAF+QACT+ND [SS] achieves the best performance of .489 and .374 in terms of accuracy and F1-macro. The [SS] model also achieves the second-best performance of .452 in terms of MAR ${ }^{\mathrm{dSet}}$. These improvements are significant over the baseline and the BIAF+QACT+ND model. Note that when using the [SS] setting, the model performance is consis- tently improved with regard to all metrics, while the accuracy of the $[\mathrm{P}+\mathrm{I}]$ model is lower than the baseline. In general, the [SS] model attains a better performance compared with the $[\mathrm{P}+\mathrm{I}]$ model despite fewer training instances. This means that when training a model using multiple corpora, it is essential to consider training instances having the same properties as our goal. Simply having more training data does not guarantee improvement.

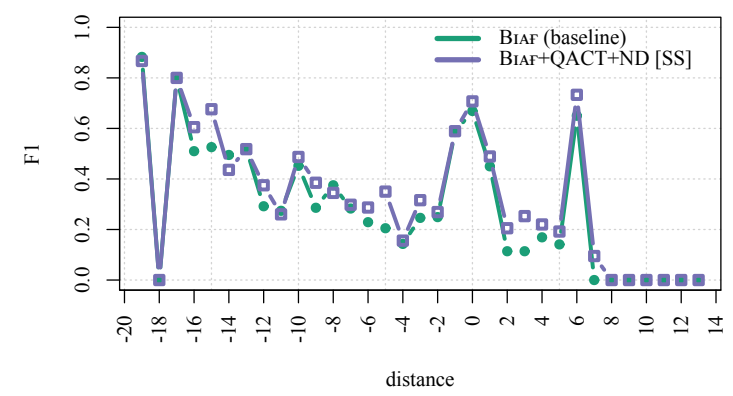

Figure 7: Model performance across distances.

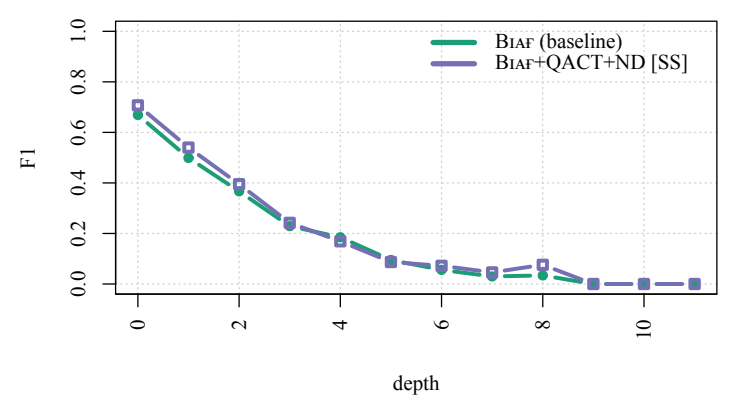

Figure 8: Model performance across depths.

To gain deeper insights into the individual link prediction improvement brought by the [SS] model over the baseline, Figure 7 shows F1 score per target linking distance. BIAF+QACT+ND [SS] is better than the baseline model, particularly at predicting short-distance links $(2 \leq|d| \leq 4$, avg. $\mathrm{F} 1=.24 \mathrm{vs} .17$ ), which is the weakest range of the baseline model. However, this is still the weakest range even for the [SS] model.

Figure 8 shows the performance across depths, that is, whether the model places each node at the proper depth in the predicted structure. $\mathrm{BIAF}+\mathrm{QACT}+\mathrm{ND}[\mathrm{SS}]$ performs better than the baseline particularly in $[0,3]$ and $[6,8]$ ranges. This plot indicates that the model performance declines as one moves further down the tree.

We next look at the models' ability to predict the role of each node (QACT task) based on the predicted hierarchical structures. Table 2 shows 


\begin{tabular}{lrrrrr}
\hline Model & Major Claim & AC (non-leaf) & AC (leaf) & Non-AC & F1-macro \\
\hline $\begin{array}{l}\text { Baseline } \\
\text { BIAF }\end{array}$ & .730 & $\underline{.639}$ & .599 & .437 & .601 \\
\hline MTL & & & & & \\
BIAF+QACT & .739 & .639 & .601 & .453 & .608 \\
BIAF+QACT+ND & .734 & .636 & .601 & .454 & .606 \\
\hline Spos & & & & & \\
BIAF+QACT+spos & .725 & .641 & .602 & .438 & .602 \\
BIAF+QACT+ND+spos & .738 & .638 & .603 & .460 & .610 \\
\hline Multi-corpora Training & & & & & \\
BIAF+QACT+ND [P+I] & $\underline{748}$ & .606 & $\mathbf{. 6 3 4 ^ { \dagger }}$ & .420 & .602 \\
BIAF+QACT+ND [SS] &. $\mathbf{7 6 7}^{\dagger}$ & .633 & .628 & $\mathbf{. 4 6 2}$ & $\mathbf{. 6 2 2}^{\dagger}$ \\
\hline
\end{tabular}

Table 2: Results for the QACT prediction. Node labels are automatically identified from the predicted topology in the main task. This table shows F1 score per node label and F1-macro. Bold-face, $\nmid$ and underline as above.

the results. The MTL models perform better compared with the baseline model. Both BIAF+QACT and BIAF+QACT+ND achieve a significant improvement over the baseline in terms of non-AC prediction and F1-macro. This reconfirms that both of our proposed MTL tasks are useful to improve linking performance. Similar to the previous result on individual links, the spos feature does not provide much improvement. We notice that the $\mathrm{BIAF}+\mathrm{QACT}+\mathrm{ND}[\mathrm{P}+\mathrm{I}]$ model performs worse than the baseline in some aspects, particularly in terms of AC (non-leaf) and non-AC predictions. On the other hand, BIAF+QACT+ ND [SS] consistently achieves better performance compared with the baseline model. It attains the highest score of .622 in F1-macro, which is a significant improvement over other configurations. This confirms our hypothesis that the difference between annotation schemes and statistical properties between PEC and ICNALE-AS2R affects the model in terms of the distribution learned. The proposed selective sampling strategy helps to alleviate this problem.

We also analyse the overall shape of the predicted structures by all models, as shown in Table 3. The gold standard trees in ICNALE-AS2R have a particular shape, expressed as the average depth of $4.3(\mathrm{SD}=1.4)$ and the leaf ratio of $.439(\mathrm{SD}=0.11)$. The baseline model tends to produce trees that are deeper and narrower than the ICNALE-AS2R gold trees. Our MTL auxiliary tasks help to improve the leaf ratio to become closer to the gold standard (significant difference between BIAF+QACT+ND and BIAF), while spos embedding does not provide additional improvement. When we introduce the multi-corpora training strategy, the predicted structures become shal-

\begin{tabular}{lrc}
\hline Model & Average Depth & Leaf Ratio \\
\hline Dataset & & \\
ICNALE-AS2R & $4.3 \pm 1.4$ & $.439 \pm .11$ \\
PEC [all] & $2.8 \pm .63$ & $.540 \pm .09$ \\
PEC [after SS] & $2.7 \pm .53$ & $.565 \pm .08$ \\
\hline Baseline & & \\
BIAF & 5.1 & .404 \\
\hline MTL & & \\
BIAF+QACT & 5.1 & .410 \\
BIAF+QACT+ND & 5.0 & .418 \\
\hline Spos & & \\
BIAF+QACT+spos & 5.2 & .407 \\
BIAF+QACT+ND+spos & 5.0 & .412 \\
\hline Multi-corpora Training & & \\
BIAF+QACT+ND [P+I] & $\underline{4.1}$ & .486 \\
BIAF+QACT+ND [SS] & $\underline{\mathbf{4 . 5}^{\dagger}}$ & $\mathbf{. 4 4 6}^{\dagger}$ \\
\hline
\end{tabular}

Table 3: Structural-output qualities of linking models. Bold-face, $\dagger$ and underline as above.

lower and wider compared with the baseline, particularly when using the $[\mathrm{P}+\mathrm{I}]$ setting. We believe this is due to the shallower trees in the PEC compared with the ICNALE-AS2R (i.e., the distributional shift problem). However, this is less of an issue for the [SS] model as it produces the most similar structure to the ICNALE-AS2R essays.

We conclude BIAF+QACT+ND [SS] as the best model in this experiment, achieving the new stateof-the-art performance for the linking task. It consistently performs better compared with other configurations across all evaluation aspects.

\subsection{Qualitative Analysis}

We conducted a qualitative analysis on some random outputs of our best model, namely BIAF+QACT+ND [SS]. Some prediction errors are likely caused by the inability of the model to re- 
solve anaphora. Figure 9 shows an output example. The model successfully groups sentences S5-S7 as a single sub-argument, as the gold annotation does. However, it connects S7 to S6, while the gold annotation connects S7 to S5 (with the support label). The link from S7 to S5 is the correct interpretation because the statement in S7 can be broadly applied to "skills of working and cooperating," not only to some specific instantiations mentioned in S6 ("set the working agenda and get help from colleagues"). A possible direction to alleviate this problem is to equip the model with a better ability to resolve anaphora. Another direction is to perform an anaphora resolution step before parsing the argumentative structure.

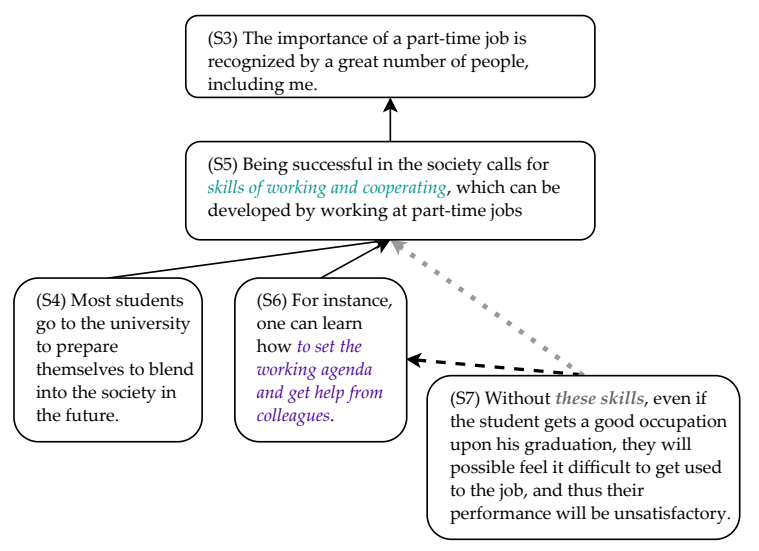

Figure 9: An excerpt of prediction for essay "W_HKG_PTJ0_029_B2_0_EDIT". The essay was written in response to a prompt "It is important for college students to have a part-time job." The dashed line illustrates an erroneous link, while the dotted line (grey) illustrates the corresponding gold link.

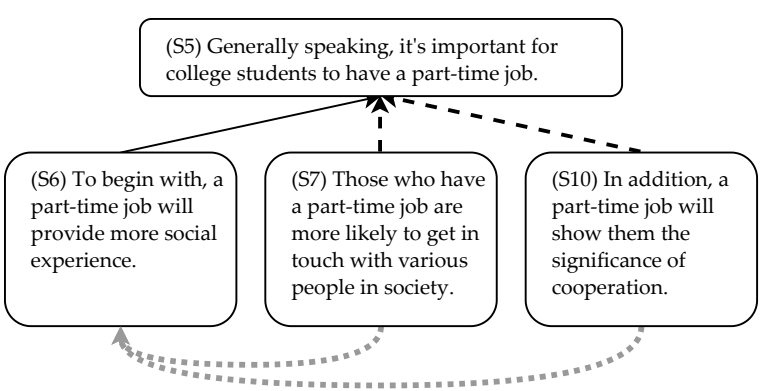

Figure 10: An excerpt of prediction for essay "W_CHN_PTJ0_242_B2_0_EDIT". The essay was written in response to a prompt "It is important for college students to have a part-time job." Dashed lines and dotted lines as above.

Another source of error is the difficulty in judging whether two statements argue at the same con- ceptual level (hence the same depth in the tree). Figure 10 shows an illustration. S7 and S10 are exemplifications of S6, and therefore, they should be placed lower than S6. However, the model recognises S6, S7 and S10 arguing at the same conceptual level. The exemplifications are probably not obvious for the model because S7 is not accompanied by a discourse marker (e.g., "for example"). In general, EFL essays are often challenging to process due to improper use (can be excessive or limited too) of discourse markers.

\section{Conclusion}

In this paper, we presented a study on the argumentative linking task; given an essay as input, a linking model outputs the argumentative structure in a tree representation. We conducted experiments using an EFL corpus, namely ICNALE-AS2R. We extended the state-of-the-art biaffine attention model using a novel set of structural auxiliary tasks in the multi-task learning setup. Additionally, we also proposed a multi-corpora training strategy using the PEC to increase training instances. It has to be noted that simply increasing the training data size does not guarantee improvement. We need to ensure that the model still learns the desired target distribution as well. To this end, we filtered PEC essays using a selective sampling technique. These two strategies provided useful supervision signals to the biaffine model and significantly improved its performance. The F1-macro for individual link predictions was boosted to .374 from .323 . Our strategies also improved the model performance on the structural aspects, achieving the MAR ${ }^{\mathrm{dset}}$ of .452 from .419, and the F1-macro of .622 from .601 for the QACT task.

A possible future direction is to evaluate whether our proposed methods are beneficial for more complex discourses, such as scientific articles. It is also necessary to provide an even richer supervision signal to improve the linking performance. One possibility is to pre-train the model on the anaphora resolution task.

\section{Acknowledgements}

This work was partially supported by Tokyo Tech World Research Hub Initiative (WRHI), JSPS KAKENHI grant number 20J13239 and Support Centre for Advanced Telecommunication Technology Research. We would like to thank anonymous reviewers for their useful and detailed feedback. 


\section{References}

Pablo Accuosto and Horacio Saggion. 2019. Transferring knowledge from discourse to arguments: A case study with scientific abstracts. In Proceedings of the 6th Workshop on Argument Mining, pages 4151, Florence, Italy. Association for Computational Linguistics.

Khalid Al-Khatib, Henning Wachsmuth, Matthias Hagen, Jonas Köhler, and Benno Stein. 2016. Crossdomain mining of argumentative text through distant supervision. In Proceedings of the 2016 Conference of the North American Chapter of the Association for Computational Linguistics: Human Language Technologies, pages 1395-1404, San Diego, California. Association for Computational Linguistics.

Kevin D. Ashley. 1990. Modeling legal argument - reasoning with cases and hypotheticals. Artificial intelligence and legal reasoning. MIT Press.

Elena Cabrio and Serena Villata. 2018. Five years of argument mining: A data-driven analysis. In Proceedings of the 27th International Joint Conference on Artificial Intelligence, IJCAI'18, page 5427-5433. AAAI Press.

Chenhui Chu, Raj Dabre, and Sadao Kurohashi. 2017. An empirical comparison of domain adaptation methods for neural machine translation. In Proceedings of the 55th Annual Meeting of the Association for Computational Linguistics (Volume 2: Short Papers), pages 385-391, Vancouver, Canada. Association for Computational Linguistics.

Y. Chu and T. Liu. 1965. On the shortest arborescence of a directed graph. Science Sinica, page 1396-1400.

Oana Cocarascu, Elena Cabrio, Serena Villata, and Francesca Toni. 2020. A dataset independent set of baselines for relation prediction in argument mining. In Proceedings of Computational Models of Argument (COMMA), pages 45-52.

Johannes Daxenberger, Steffen Eger, Ivan Habernal, Christian Stab, and Iryna Gurevych. 2017. What is the essence of a claim? cross-domain claim identification. In Proceedings of the 2017 Conference on Empirical Methods in Natural Language Processing, pages 2055-2066, Copenhagen, Denmark. Association for Computational Linguistics.

Timothy Dozat and Christopher D. Manning. 2017 Deep biaffine attention for neural dependency parsing. In Proceedings of the International Conference on Learning Representations (ICLR).

Jack Edmonds. 1967. Optimum branchings. Journal of Research of the National Bureau of Standards - B. Mathematics and Mathematical Physics, 71B:233240.

Steffen Eger, Johannes Daxenberger, and Iryna Gurevych. 2017. Neural end-to-end learning for computational argumentation mining. In Proceedings of the 55th Annual Meeting of the Association for Computational Linguistics (Volume 1: Long Papers), pages 11-22, Vancouver, Canada. Association for Computational Linguistics.

Steven Y. Feng, Varun Gangal, Jason Wei, Sarath Chandar, Soroush Vosoughi, Teruko Mitamura, and Eduard H. Hovy. 2021. A survey of data augmentation approaches for NLP. CoRR, abs/2105.03075.

Yumi Fujiwara. 2018. The role of grammar instruction in japanese efl context: Towards communicative language teaching. Journal of the Academic Society for Quality of Life (JAS4QoL), 4(4):1-11.

Matt Gardner, Joel Grus, Mark Neumann, Oyvind Tafjord, Pradeep Dasigi, Nelson F. Liu, Matthew E. Peters, Michael Schmitz, and Luke Zettlemoyer. 2018. Allennlp: A deep semantic natural language processing platform. CoRR, abs/1803.07640.

Shinichiro Ishikawa. 2013. The icnale and sophisticated contrastive interlanguage analysis of asian learners of english. Learner Corpus Studies in Asia and the World, 1:91-118.

Shinichiro Ishikawa. 2018. The icnale edited essays: A dataset for analysis of 12 english learner essays based on a new integrative viewpoint. English Corpus Linguistics, 25:1-14.

Ann M. Johns. 1986. The esl student and the revision process: Some insights from scheme theory. Journal of Basic Writing, 5(2):70 - 80.

Robert B. Kaplan. 1966. Cultural thought patterns in inter-cultural education. Language Learning, 16(12):1-20.

Diederik P. Kingma and Jimmy Lei Ba. 2015. Adam: a method for stochastic optimization. In Proceedings of International Conference on Learning Representations (ICLR).

Anne Lauscher, Goran Glavaš, Simone Paolo Ponzetto, and Kai Eckert. 2018. Investigating the role of argumentation in the rhetorical analysis of scientific publications with neural multi-task learning models. In Proceedings of the 2018 Conference on Empirical Methods in Natural Language Processing, pages 3326-3338, Brussels, Belgium. Association for Computational Linguistics.

John Lawrence and Chris Reed. 2020. Argument Mining: A Survey. Computational Linguistics, 45(4):765-818.

Marco Lippi and Paolo Torroni. 2016. Argumentation mining: State of the art and emerging trends. $A C M$ Trans. Internet Technol., 16(2).

Pei Liu, Xuemin Wang, Chao Xiang, and Weiye Meng. 2020. A survey of text data augmentation. In 2020 International Conference on Computer Communication and Network Security (CCNS), pages 191-195. 
Gaku Morio, Hiroaki Ozaki, Terufumi Morishita, Yuta Koreeda, and Kohsuke Yanai. 2020. Towards better non-tree argument mining: Proposition-level biaffine parsing with task-specific parameterization. In Proceedings of the 58th Annual Meeting of the Association for Computational Linguistics, pages 3259-3266, Online. Association for Computational Linguistics.

Eric W. Noreen. 1989. Computer Intensive Methods for Testing Hypotheses: An Introduction. Wiley, New York.

Joonsuk Park and Claire Cardie. 2018. A corpus of eRulemaking user comments for measuring evaluability of arguments. In Proceedings of the Eleventh International Conference on Language Resources and Evaluation (LREC 2018), Miyazaki, Japan. European Language Resources Association (ELRA).

Adam Paszke, Sam Gross, Francisco Massa, Adam Lerer, James Bradbury, Gregory Chanan, Trevor Killeen, Zeming Lin, Natalia Gimelshein, Luca Antiga, Alban Desmaison, Andreas Kopf, Edward Yang, Zachary DeVito, Martin Raison, Alykhan Tejani, Sasank Chilamkurthy, Benoit Steiner, Lu Fang, Junjie Bai, and Soumith Chintala. 2019. Pytorch: An imperative style, high-performance deep learning library. In H. Wallach, H. Larochelle, A. Beygelzimer, F. d'Alché-Buc, E. Fox, and R. Garnett, editors, Advances in Neural Information Processing Systems 32, pages 8024-8035. Curran Associates, Inc.

Andreas Peldszus and Manfred Stede. 2015. Joint prediction in MST-style discourse parsing for argumentation mining. In Proceedings of the 2015 Conference on Empirical Methods in Natural Language Processing, pages 938-948, Lisbon, Portugal. Association for Computational Linguistics.

Andreas Peldszus and Manfred Stede. 2016. An annotated corpus of argumentative microtexts. In Argumentation and Reasoned Action - Proceedings of the 1st European Conference on Argumentation, Lisbon, 2015.

Peter Potash, Alexey Romanov, and Anna Rumshisky. 2017. Here's my point: Joint pointer architecture for argument mining. In Proceedings of the 2017 Conference on Empirical Methods in Natural Language Processing, pages 1364-1373, Copenhagen, Denmark. Association for Computational Linguistics.

Jan Wira Gotama Putra, Simone Teufel, and Takenobu Tokunaga. 2021a. Annotating argumentative structure in english-as-foreign-language learner essays. Natural Language Engineering, pages 1-37.

Jan Wira Gotama Putra, Simone Teufel, and Takenobu Tokunaga. 2021b. Parsing argumentative structure in English-as-foreign-language essays. In Proceedings of the 16th Workshop on Innovative Use of NLP for Building Educational Applications, pages 97109, Online. Association for Computational Linguistics.

Ella Rabinovich, Sergiu Nisioi, Noam Ordan, and Shuly Wintner. 2016. On the similarities between native, non-native and translated texts. In Proceedings of the 54th Annual Meeting of the Association for Computational Linguistics (Volume 1: Long Papers), pages 1870-1881, Berlin, Germany. Association for Computational Linguistics.

Nils Reimers and Iryna Gurevych. 2019. SentenceBERT: Sentence embeddings using Siamese BERTnetworks. In Proceedings of the 2019 Conference on Empirical Methods in Natural Language Processing and the 9th International Joint Conference on Natural Language Processing (EMNLP-IJCNLP), pages 3982-3992.

Ramon Ruiz-Dolz, Jose Alemany, Stella Heras, and Ana Garcia-Fornes. 2021. Transformer-based models for automatic identification of argument relations: A cross-domain evaluation. IEEE Intelligent Systems, pages 1-1.

Claudia Schulz, Steffen Eger, Johannes Daxenberger, Tobias Kahse, and Iryna Gurevych. 2018. Multi-task learning for argumentation mining in low-resource settings. In Proceedings of the 2018 Conference of the North American Chapter of the Association for Computational Linguistics: Human Language Technologies, Volume 2 (Short Papers), pages 35-41, New Orleans, Louisiana. Association for Computational Linguistics.

Tony Silva. 1993. Toward an understanding of the distinct nature of 12 writing: The esl research and its implications. TESOL Quarterly, 27(4):657-677.

Wei Song, Ziyao Song, Ruiji Fu, Lizhen Liu, Miaomiao Cheng, and Ting Liu. 2020. Discourse selfattention for discourse element identification in argumentative student essays. In Proceedings of the 2020 Conference on Empirical Methods in Natural Language Processing (EMNLP), pages 2820-2830, Online. Association for Computational Linguistics.

Christian Stab and Iryna Gurevych. 2014. Identifying argumentative discourse structures in persuasive essays. In Proceedings of the 2014 Conference on Empirical Methods in Natural Language Processing $(E M N L P)$, pages 46-56, Doha, Qatar. Association for Computational Linguistics.

Christian Stab and Iryna Gurevych. 2017. Parsing argumentation structures in persuasive essays. Computational Linguistics, 43(3):619-659.

Christian Stab, Tristan Miller, Benjamin Schiller, Pranav Rai, and Iryna Gurevych. 2018. Crosstopic argument mining from heterogeneous sources. In Proceedings of the 2018 Conference on Empirical Methods in Natural Language Processing, pages 3664-3674, Brussels, Belgium. Association for Computational Linguistics. 
Yuxiao Ye and Simone Teufel. 2021. End-to-end argument mining as biaffine dependency parsing. In Proceedings of the 16th Conference of the European Chapter of the Association for Computational Linguistics: Main Volume, pages 669-678, Online. Association for Computational Linguistics.

\section{A Implementation Notes}

Hidden Units and Model Implementation 512 units for the first dense layer, 256 LSTM unit, and 256 units for the second dense layer after the BiLSTM layer. Dropout is applied between all layers. For a fair comparison, we follow Putra et al. (2021b) in using the sentence-BERT encoder fine-tuned on the NLI dataset ("bert-base-nli-meantokens", https://github.com/UKPLab/ sentence-transformers). All models are trained using Adam optimiser (Kingma and Ba, 2015), and implemented in PyTorch (Paszke et al., 2019) and AllenNLP (Gardner et al., 2018).

Loss The MTL loss is defined as $L=$ $\sum_{t} \frac{1}{2 \sigma_{t}^{2}} L_{t}+\ln \left(\sigma_{t}\right)$, where the loss $L_{t}$ of each task $t$ is dynamically weighted, controlled by a learnable parameter $\sigma_{t}$. The loss for the main task (sentence linking) is computed using max-margin criterion, while losses for auxiliary tasks are computed using cross-entropy. 